\title{
On some universal construction of minimal topological generating sets for inverse limits of iterated wreath products of non-Abelian finite simple groups
}

\author{
Adam Woryna
}

Received: 12 June 2013 / Accepted: 10 February 2015 / Published online: 24 February 2015

C The Author(s) 2015. This article is published with open access at Springerlink.com

\begin{abstract}
Let $\left(H_{i}\right)_{i \geq 1}$ be an arbitrary sequence of non-Abelian finite simple transitive permutation groups. By using the combinatorial language of time-varying automata, we provide an explicit and naturally defined construction of a two-element set which generates a dense subgroup in the inverse limit ... $2 \mathrm{H}_{2} 2 \mathrm{H}_{1}$ of iterated permutational wreath products of the groups $H_{i}$. The corresponding automaton is equipped with three states, one of which is neutral and the semigroup generated by the other two states is free. We derive other algebraic and geometric properties of the group generated by this automaton. By using the notion of a Mealy automaton, we obtain the analogous construction for the infinite permutational wreath power of an arbitrary non-Abelian finite simple transitive permutation group $H$ on a set $X$. We show that the wreath power ... $2 H<H$ contains a dense 2-generated not finitely presented amenable subgroup of exponential growth, which is generated by a 3-state Mealy automaton over the alphabet $X$. The self-similar group generated by this automaton is self-replicating, contracting and regular weakly branch over the commutator subgroup.
\end{abstract}

Keywords Wreath product - Automorphisms of rooted trees - Mealy automaton Time-varying automaton · Group generated by an automaton

Mathematics Subject Classification $20 B 27 \cdot 20 B 40 \cdot 20$ F29 $\cdot$ 20E22 - 20E18 . $20 \mathrm{E} 08$

\footnotetext{
A. Woryna $(\varangle)$

Institute of Mathematics, Silesian University of Technology, ul. Kaszubska 23,

44-100 Gliwice, Poland

e-mail: adam.woryna@polsl.pl
} 


\section{Introduction and main results}

Let $X$ be an arbitrary changing alphabet, i.e., an infinite sequence $\left(X_{i}\right)_{i \geq 1}=$ $\left(X_{1}, X_{2}, \ldots\right)$ of non-empty finite sets (sets of letters) and let $X^{*}$ be the rooted tree defined by $X$. The tree $X^{*}$ consists of finite words which are sequences from finite cartesian products of the form $X^{t}:=X_{1} \times \cdots \times X_{t}(t \geq 1)$ including the empty sequence as a root. The automorphism group $A u t\left(X^{*}\right)$ is an example of a topological group equipped with its natural profinite topology induced by a metric in which two elements $f, g \in \operatorname{Aut}\left(X^{*}\right)$ are close if they act in the same way on the set $X^{t}$ for a large value of $t$ (see Sect. 2). Let $H_{i}(i \geq 1)$ be a transitive permutation group on the set $X_{i}$. In the present paper, we consider the corresponding inverse limit

$$
W_{\infty}=\ldots \text {. } H_{2} \prec H_{1}
$$

of iterated permutational wreath products of the groups $H_{i}$ as a closed subgroup in the group $A u t\left(X^{*}\right)$ which consists of all automorphisms $g$ such that for every vertex $w \in X^{*}$, the restriction of $g$ to the subtree $\left\{w x: x \in X_{|w|+1}\right\} \subseteq X^{*}$ (the so-called label of $g$ at the vertex $w$, denoted further by $g(w)$ ) coincides with some element from $H_{|w|+1}$, where $|w|$ denotes the length of a word $w$. According to [22] if each $H_{i}$ is a non-Abelian simple transitive group, then $W_{\infty}$ is topologically 2 -generated. The methods used in [22] are of probabilistic nature and do not provide any explicit construction of a finite topological generating set for $W_{\infty}$. On the other hand, there are such constructions in the particular cases of the groups $H_{i}$. They turned out to be fruitful in providing interesting properties of the groups generated by these sets, or the corresponding inverse limits. For example, in the case when each $H_{i}(i \geq 1)$ is the alternating group $A_{n_{i}}$ of degree $n_{i} \geq 5$ with its natural action on the set $X_{i}=\left\{1,2, \ldots, n_{i}\right\}$, a finite topological generating set was constructed by Bhattacharjee [4] to show that the corresponding inverse limit ... $2 A_{n_{2}} 2 A_{n_{1}}$ is generated by 2 elements even with positive probability.

Definition 1 A generating set of a group $G$ (topological generating set if $G$ is a closed subgroup of a topological group) is called minimal if it contains exactly $d(G)$ elements, where $d(G)$ denotes the (topological) rank of $G$, i.e., the minimal number of elements in a (topological) generating set.

The generating set from [4] is minimal; however, its inductive description, which is based on some specific generators of alternating groups $A_{n_{i}}$ depending on the divisibility by 4 of the degree $n_{i}$, seems to be complex and difficult in handling. More convenient to study is the recent example due to Brieussel [6] providing a minimal topological generating set for the group ...2 $A_{n_{2}} 2 A_{n_{1}}$, where the two generators are of finite order and generate a group of intermediate growth. This construction originates from the concept of Wilson [26] of the so-called eligible pairs of elements in the alternating groups $A_{n_{i}}$, and it assumes that $n_{i} \geq 29$ for every $i \geq 1$. In [31], we constructed a minimal topological generating set for infinite wreath powers . . 2 $\mathrm{H} 2 \mathrm{H}$, where $H$ is a finite transitive perfect group generated by 2 elements one of which has a fixed point and the other decomposes into disjoint cycles of the same length. In [31], we realized the generating set by a 3-state Mealy automaton and showed that we can 
take as $H$ the alternating group of degree $n \geq 5$ or the projective special linear group $P S L_{2}(p)$ with any prime $p>3$. In some particular cases of the groups $H_{i}$, certain finite topological generating sets for the inverse limit (1) can also be obtained by using the notions of rooted and directed automorphisms. These generating sets are not minimal, but they provide known examples of finitely generated branch groups- the concept introduced by Grigorchuk in [13] and used by Bartholdi [1], Bondarenko [5], Gupta and Sidki [15], Segal [23], and others to discover extraordinary properties of these groups.

In this paper, we introduce a different construction of automorphisms, which provides minimal topological generating sets for inverse limits of the form (1). Our construction, in contrast to the above mentioned, is universal in the sense that it works with an arbitrary sequence $\left(H_{i}\right)_{i \geq 1}$ of non-Abelian finite simple transitive permutation groups. We base it on the observation that for each $i \geq 1$, there is a triple

$$
\left(\alpha_{i}, \beta_{i}, x_{i}\right) \in H_{i} \times H_{i} \times X_{i}
$$

(further called a hooked triple of $H_{i}$ ) such that the following two conditions hold:

- the permutations $\alpha_{i}, \beta_{i}$ generate the group $H_{i}$,

- there are two non-trivial cycles in decompositions of $\alpha_{i}$ and $\beta_{i}$ into the products of disjoint cycles, the first cycle from the decomposition of $\alpha_{i}$ and the second cycle from the decomposition of $\beta_{i}$, such that $x_{i}$ is the only element which belongs to the both supports of these cycles.

If the number of letters in the set $X_{i}$ is even, then the existence of such a triple directly follows from the result settled definitely by Stein [24] and concerning the socalled $1 \frac{1}{2}$-generation of non-Abelian finite simple groups. If $\left|X_{i}\right|$ is odd, we use FeitJones $[11,17]$ results classifying all finite primitive permutation groups containing a regular cyclic subgroup. Next, we define two automorphisms $a, b \in \operatorname{Aut}\left(X^{*}\right)$ such that the labels of both $a$ and $b$ at any word $w \in X^{*}$ are trivial, unless $w$ is a beginning (maybe empty) of the infinite sequence $x_{1} x_{2} \ldots \in \partial X^{*}$ of letters from the above hooked triples and in this case we take: $a(w)=\alpha_{|w|+1}, b(w)=\beta_{|w|+1}$.

Further, we find it convenient to use the combinatorial language of a time-varying automaton and define the group generated by the set $\{a, b\}$ as an automaton group generated by a 3 -state time-varying automaton $\mathcal{A}$ with the set

$$
Q=\{a, b, i d\}
$$

as a set of states, where $i d$ denotes the identity automorphism of the corresponding tree. We define the automaton $\mathcal{A}$ by the following system of wreath recursions of the so-called $i$-th transitions $a_{i}, b_{i}(i \geq 1)$ of the states:

$$
\left\{\begin{array}{l}
a_{i}=\left(a_{i+1}, i d, \ldots, i d\right) \alpha_{i}, \\
b_{i}=\left(b_{i+1}, i d, \ldots, i d\right) \beta_{i},
\end{array} \quad i \geq 1 .\right.
$$

In above equations, we assume an ordering of letters in the set $X_{i}(i \geq 1)$ such that the letter $x_{i}$ occurs in the first position. We obtain the following 
Theorem 1 The inverse limit $W_{\infty}=\ldots 2 H_{2} \prec H_{1}$ is topologically generated by the time-varying automaton $\mathcal{A}$ defined by (2)-(3).

In the regular case, we deal with an infinite wreath power ... $2 H_{2} H$ of an arbitrary non-Abelian finite simple transitive permutation group $H$ on a set $X$ and we take an arbitrary hooked triple

$$
(\alpha, \beta, x) \in H \times H \times X
$$

of the group $H$. In this case, the above construction brings a Mealy automaton over the alphabet $X$ with the set of states (2) and the following system of wreath recursions:

$$
\left\{\begin{array}{l}
a=(a, i d, \ldots, i d) \alpha, \\
b=(b, i d, \ldots, i d) \beta .
\end{array}\right.
$$

Corollary 1 The infinite wreath power ... $2 \mathrm{H} 2 \mathrm{H}$ is topologically generated by the Mealy automaton with the set of states (2) and the wreath recursions (4).

Further, we derive some algebraic and geometric properties of the group generated by the time-varying automaton $\mathcal{A}$ defined by (2)-(3). In Sect. 3, we introduce the notion of a nearly finitary automorphism and a nearly finitary group of automorphisms. Namely, an automorphism $g \in \operatorname{Aut}\left(X^{*}\right)$ is called nearly finitary if there is a finite subset $U$ (perhaps empty) of the boundary $\partial X^{*}$ and a number $n$ such that for every word $w \in X^{*}$ of length $|w| \geq n$ which is not a beginning of any sequence from $U$ the label $g(w)$ is trivial and for every sequence $u \in U$ we have $u^{g} \neq u$. We call a subgroup of $A u t\left(X^{*}\right)$ in which every element is a nearly finitary automorphism a nearly finitary group. Every nearly finitary automorphism is an example of a bounded automorphism (for the definition of a bounded automorphism see [21]). Nekrashevych showed ([21], Theorem 4.4) that if the tree $X^{*}$ has a bounded degree of vertices, then the group of bounded automorphisms does not contain non-Abelian free subgroups. In the present paper, by using the characterization of groups acting faithfully on rooted trees due to Nekrashevych ([21], Theorem 3.3), we show that in the case of nearly finitary groups, there is no need for the above restriction on the degree of vertices and we obtain the following:

Proposition 1 Let $X=\left(X_{i}\right)_{i \geq 1}$ be an arbitrary changing alphabet (with a bounded or unbounded degree of vertices) and let $G \leq A u t\left(X^{*}\right)$ be a nearly finitary group. Then $G$ does not contain non-Abelian free subgroups.

In Sect. 6, we introduce the notion of an automaton with a nucleus as well as the notion of an automaton with a path-active nucleus and show (Proposition 4) that every automaton with a path-active nucleus generates a nearly finitary group. In Proposition 8 , we show that the automaton $\mathcal{A}$ is an automaton with a path-active nucleus, which gives the following result.

Theorem 2 The group $G(\mathcal{A})=\langle a, b\rangle$ generated by the automaton $\mathcal{A}$ does not contain non-Abelian free subgroups. 
On the other hand, we see that the semigroup generated by the states $a, b \in Q$ of the automaton $\mathcal{A}$ is free (Proposition 9).

In Sect. 5, we introduce the notion of a disappearing group word of a time-varying automaton $A$ and the notion of the depth of a disappearing group word. Every disappearing group word defines the neutral element in the group $G(A)$ generated by the automaton $A$. We consider the case when the set of disappearing group words coincides with the set of all group words defining the neutral element. In this case, we show that if the depths of disappearing group words are not uniformly bounded, then the group $G(A)$ is not finitely presented. The automaton $\mathcal{A}$, as we show, satisfies both these conditions, and hence, we obtain the following

Theorem 3 The group $G(\mathcal{A})$ is not finitely presented.

The case when every group word defining the neutral elemental is a disappearing group word was also studied by Erschler [10], where the author restricted the study to a fixed alphabet and called the corresponding groups as piecewise automatically presented groups. In [10], A. Erschler showed that if such a group satisfies the property T of Kazhdan, then it must be finite and if this is an amenable finitely presented group, then it must be virtually Abelian.

In the present paper, we also derive some geometric properties of the group $G(\mathcal{A})$ related to fractalness, branches and contraction. Namely, in Sect. 4, we introduce the notion of a self-replicating time-varying automaton as well as the notion of a branching automaton $A$ over an infinite sequence $\left(K_{i}\right)_{i \geq 1}$ of subgroups $K_{i} \leq G\left(A_{i}\right)$, where $G\left(A_{i}\right)$ is the group generated by the $i$-th transition of $A$. These notions constitute some natural generalization of the concepts of a self-replicating group (sometimes called a fractal group) and a regular weakly branch group, which are well known in branch group theory and concern the so-called self-similar groups acting on regular rooted trees. Also, the item (i) in Definition 12 means that every Mealy automaton $A$ which is an automaton with a nucleus generates a self-similar contracting group (for the corresponding definitions see, for example, [2] or [20]). We derive the following

Theorem 4 The automaton $\mathcal{A}$ is self-replicating and branching over the sequence $\left(K_{i}\right)_{i \geq 1}$ of commutator subgroups $K_{i}=G\left(\mathcal{A}_{i}\right)^{\prime}$. In particular, the group $G(\mathcal{A})$ is weakly branch and in the regular case, this is a self-replicating contracting group which is regular weakly branch over the commutator subgroup.

Note that in the regular case, our construction gives a bounded Mealy automaton. Due to the main result in [3], the groups generated by bounded automata are amenable. Consequently, our construction gives the following

Corollary 2 The infinite permutational wreath power ... 2 $\mathrm{H} 2 \mathrm{H}$ of an arbitrary nonAbelian finite simple transitive permutation group $H$ on a set $X$ contains a dense 2-generated not finitely presented amenable subgroup of exponential growth, which is generated by a 3-state Mealy automaton over the alphabet X. The group generated by this automaton is self-replicating, contracting and regular weakly branch over the commutator subgroup. 


\section{The tree $X^{*}$ and the group $\operatorname{Aut}\left(X^{*}\right)$}

The tree $X^{*}$ of finite words over a changing alphabet $X=\left(X_{i}\right)_{i \geq 1}$ consists of all finite sequences of the form $x_{1} x_{2} \ldots x_{t}(t \geq 1)$, where $x_{i} \in X_{i}$ for $i=1, \ldots, t$. For a given $t \geq 1$, we obtain the set $X^{t}=\left\{w \in X^{*}:|w|=t\right\}$ of words of the length $t$. We also include the empty word $\epsilon$ of zero length. In the tree $X^{*}$, two words are adjacent if and only if one of these words is obtained by adding a single letter to the end of the other word. Hence, we see that $X^{*}$ is a spherically homogeneous rooted tree with the empty word $\epsilon$ as a root and the set $X^{t}$ as a $t$-th level $(t \geq 0)$, i.e., the set of vertices with the distance $t$ from the root. By $X_{(i)}^{*}(i \geq 1)$, we denote the tree of finite words over the changing alphabet $X_{(i)}=\left(X_{i+j}\right)_{j \geq 0}$. In particular, we have: $X^{*}=X_{(1)}^{*}$. If $w \in X_{(i)}^{*}$ and $v \in X_{(i+|w|)}^{*}$ for some $i \geq 1$, then we denote by $w v$ the concatenation of the words $w, v$; obviously $w v \in X_{(i)}^{*}$.

We will also consider the boundary $X^{\omega}=\partial X^{*}$ of the tree $X^{*}$, i.e., the set of all infinite paths starting at the root: $X^{\omega}=\left\{x_{1} x_{2} \ldots: x_{i} \in X_{i}, i \geq 1\right\}$.

If $\left(X_{i}\right)_{i \geq 1}$ is a constant sequence, then we identify the changing alphabet $X$ with the set $X_{1}$ and call a fixed alphabet. In this case, we obtain the so-called regular rooted tree.

The group $A u t\left(X^{*}\right)$ of automorphisms of the tree $X^{*}$ is a subgroup in the symmetric group $\operatorname{Sym}\left(X^{*}\right)$ on the set of vertices which consists of all permutations that preserve the adjacency relation in the tree $X^{*}$. Directly from this definition, one can see that given an automorphism $g \in A u t\left(X^{*}\right)$ and a word $w \in X^{*}$ with $|w|=i(i \geq 0)$, there is a unique automorphism $h \in \operatorname{Aut}\left(X_{(i+1)}^{*}\right)$ which satisfies: $(w v)^{g}=w^{g} v^{h}$ for every $v \in X_{(i+1)}^{*}$. We denote this automorphism by $g_{\{w\}}$ and call it the section of the automorphism $g$ at the word $w$. By $g(w)$, we denote the restriction of the section $g_{\{w\}}$ to the set of one-letter words and call this the label of the automorphism $g$ at the word $w$. Obviously, we have: $g(w) \in \operatorname{Sym}\left(X_{i+1}\right)$. The label of the automorphism $g$ at the empty word is called the root permutation of $g$. Note that the labels $g(w), w \in X^{*}$ of an automorphism $g \in \operatorname{Aut}\left(X^{*}\right)$ define $g$ uniquely, as for any word $w=x_{1} x_{2} \ldots x_{t}$ we have

$$
w^{g}=x_{1}^{g(\epsilon)} x_{2}^{g\left(x_{1}\right)} x_{3}^{g\left(x_{1} x_{2}\right)} \ldots x_{t}^{g\left(x_{1} \ldots x_{t-1}\right)} .
$$

Note that the above formulas also allow to define in a natural way the action of any $g \in \operatorname{Aut}\left(X^{*}\right)$ on the boundary $X^{\omega}$.

For every $i \geq 1$, let us fix an ordering of letters in the set $X_{i}$ :

$$
X_{i}=\left\{x_{i, 1}, x_{i, 2}, \ldots, x_{i, m_{i}}\right\}
$$

Then the mapping

$$
g \mapsto\left(\left(g_{\left\{x_{i, 1}\right\}}, \ldots, g_{\left\{x_{i, m_{i}}\right\}}\right), g(\epsilon)\right),
$$

defines an isomorphism of the group $\operatorname{Aut}\left(X_{(i)}^{*}\right)$ with the permutational wreath product $\operatorname{Aut}\left(X_{(i+1)}^{*}\right)$ 2 $\operatorname{Sym}\left(X_{i}\right)$, which, by definition, is the semidirect product 


$$
\operatorname{Aut}\left(X_{(i+1)}^{*}\right)^{m_{i}} \rtimes \operatorname{Sym}\left(X_{i}\right)
$$

with the action of the symmetric group $\operatorname{Sym}\left(X_{i}\right)$ on the corresponding direct power by permutation of the factors. From this isomorphism, we see that the group Aut $\left(X^{*}\right)$ is a profinite group equipped with its natural profinite topology and it can be identified with the inverse limit of iterated permutational wreath products of symmetric groups on the corresponding sets of letters:

$$
\operatorname{Aut}\left(X^{*}\right)=\ldots 2 \operatorname{Sym}\left(X_{2}\right) \text { r Sym }\left(X_{1}\right)
$$

In general, given a sequence $\left(H_{i}\right)_{i \geq 1}$ of permutation groups on the sets $X_{i}$, we consider the closed subgroup $W_{\infty}$ of $\operatorname{Aut}\left(X^{*}\right)$ that consists of all automorphisms $g \in \operatorname{Aut}\left(X^{*}\right)$ satisfying: $g(w) \in H_{|w|+1}$ for all $w \in X^{*}$; we identify $W_{\infty}$ with the inverse limit of iterated permutational wreath products of the groups $H_{i}$ :

$$
W_{\infty}=\ldots \text {. } H_{2} \text { 乙 } H_{1}
$$

We usually write the isomorphism (5) in the form

$$
g=\left(g_{\left\{x_{i, 1}\right\}}, \ldots, g_{\left\{x_{i, m_{i}}\right\}}\right) g(\epsilon)
$$

and call this equality the wreath recursion of the automorphism $g$. Note that if $h \in$ $\operatorname{Aut}\left(X_{(i)}^{*}\right)$ is an automorphism with the wreath recursion

$$
h=\left(h_{\left\{x_{i, 1}\right\}}, \ldots, h_{\left\{x_{i, m_{i}}\right\}}\right) h(\epsilon)
$$

then the wreath recursion of the product $g h$ satisfies:

$$
g h=\left(g_{\left\{x_{i, 1}\right\}} h_{\left\{z_{1}\right\}}, \ldots, g_{\left\{x_{i, m_{i}}\right\}} h_{\left\{z_{m_{i}}\right\}}\right) g(\epsilon) h(\epsilon),
$$

where $z_{r}=x_{i, r}^{g}$ for $1 \leq r \leq m_{i}$. In particular, for the wreath recursion of the inverse to $g$, we have

$$
g^{-1}=\left(\left(g_{\left\{y_{1}\right\}}\right)^{-1}, \ldots,\left(g_{\left\{y_{m_{i}}\right\}}\right)^{-1}\right)(g(\epsilon))^{-1},
$$

where $y_{r}=x_{i, r}^{g^{-1}}\left(1 \leq r \leq m_{i}\right)$.

Remark 1 Further, to simplify notation, we use the common notation and write $i d$ for the identity permutation of an arbitrary non-empty set. Also, if $g(\epsilon)=i d$, we simply write $g=\left(g_{\left\{x_{i, 1}\right\}}, \ldots, g_{\left\{x_{i, m_{i}}\right\}}\right)$. Similarly, if $g_{\{x\}}=i d$ for every $x \in X_{i}$, then we identify $g$ with the root permutation $g(\epsilon)$ and write $g=g(\epsilon)$.

For every $i \geq 1$ and every subgroup $G \leq \operatorname{Aut}\left(X_{(i)}^{*}\right)$, let us denote by $S t_{G}(1)$ the first-level stabilizer in $G$, i.e., the subgroup of $G$ which consists of automorphisms $g$ 
such that the root permutation $g(\epsilon)$ is trivial. This subgroup is normal in $G$, and it has a finite index and the mapping

$$
\Psi_{i}: S t_{G}(1) \rightarrow\left(\operatorname{Aut}\left(X_{(i+1)}^{*}\right)\right)^{m_{i}}, \quad \Psi_{i}(g)=\left(g_{\left\{x_{i, 1}\right\}}, \ldots, g_{\left\{x_{i, m_{i}}\right\}}\right)
$$

is a homomorphism. For every $1 \leq r \leq m_{i}$, the homomorphism $\Psi_{i}$ defines the canonical projection $\Phi_{i, r}$ on the $r$-th coordinate:

$$
\Phi_{i, r}: S t_{G}(1) \rightarrow \operatorname{Aut}\left(X_{(i+1)}^{*}\right), \quad \Phi_{i, r}(g)=g_{\left\{x_{i, r}\right\}} .
$$

\section{Nearly finitary automorphisms and nearly finitary groups}

Definition 2 An automorphism $g \in \operatorname{Aut}\left(X^{*}\right)$ is called nearly finitary if there is a finite subset $U \subseteq \partial X^{*}$ (perhaps empty) and a number $n$ such that for every word $w \in X^{*}$ of length $|w| \geq n$ which is not a beginning of any sequence from $U$, the label $g(w)$ is trivial and for every sequence $u \in U$, we have: $u^{g} \neq u$.

Note that the inverse of a nearly finitary automorphism is a nearly finitary automorphism. Directly by the above definition, we also see that if $g \in A u t\left(X^{*}\right)$ is a nearly finitary automorphism and $u \in \partial X^{*}$ is an infinite path such that the labels of $g$ are not trivial for infinitely many beginnings of $u$, then $u$ cannot be a fixed point of $g$. This implies that the composition of two nearly finitary automorphisms is not necessarily a nearly finitary automorphism. Indeed, let $X=\{1,2,3\}$ be a fixed alphabet and let us define the automorphism $a \in A u t\left(X^{*}\right)$ by its wreath recursion as follows: $a=(i d, i d, a)(1,2)$. Then the infinite path $3^{\infty}=33 \ldots$ is a fixed point of $a$, and for every $i \geq 1$, the label $a\left(3^{i}\right)$ is equal to the transposition $(1,2)$. Hence, the automorphism $a$ is not nearly finitary, but $a$ is the composition of the automorphisms $b, c \in \operatorname{Aut}\left(X^{*}\right)$, where $b=(i d, i d, b)(1,3)$ and $c=(c, i d, i d)(1,3,2)$. We verify directly by the definition that the automorphisms $b$ and $c$ are nearly finitary.

In the present paper, we provide naturally defined examples of groups which have the property that every element of such a group is a nearly finitary automorphism of the corresponding rooted tree.

Definition 3 A subgroup of the group $\operatorname{Aut}\left(X^{*}\right)$ of which every element is a nearly finitary automorphism of the tree $X^{*}$ is called a nearly finitary group.

Below, we recall some terminology and results due to Nekrashevych [21]. We use them to prove that nearly finitary groups do not contain non-Abelian free subgroups.

Theorem 5 ([21], Theorem 3.3) Let $G$ be a group acting faithfully on an infinite locally finite one-rooted tree $T$. Then one of the following holds.

(1) G has no free non-Abelian subgroups,

(2) there is $u \in \partial T$ and a free non-Abelian subgroup $F \leq G$ which is contained in the stabilizer of $u$ and such that $F$ acts faithfully on the neighborhood $w X_{(|w|+1)}^{\omega}$ of $u$, where $w \in T$ is an arbitrary beginning of the sequence $u$,

(3) there is $u \in \partial T$ and a free non-Abelian subgroup $F \leq G$ such that the stabilizer of $u$ in the group $F$ is trivial. 
Let $X=\left(X_{i}\right)_{i \geq 1}$ be a changing alphabet and let $X^{*}$ be the corresponding rooted tree. A subtree $T \subseteq X^{*}$ containing the root of $X^{*}$ is called a rooted subtree of $X^{*}$. Let $L_{n}=T \cap X^{n}$ be the $n$-th level of the tree $T$. An automorphism $g \in \operatorname{Aut}\left(X^{*}\right)$ is said to be almost finitary on $T$ if

$$
\lim _{n \rightarrow \infty} \frac{\theta_{g, T}(n)}{\left|L_{n}\right|}=0
$$

where $\theta_{g, T}(n)$ is the number of all vertices $w \in L_{n}$ such that the section $g_{\{w\}}$ is a non-trivial automorphism of the tree $X_{(|w|+1)}^{*}$.

Definition 4 A locally finite graph is said to be amenable if for every $\epsilon>0$, there is a finite set $F_{\epsilon}$ of vertices such that $\left|\partial F_{\epsilon}\right| /\left|F_{\epsilon}\right|<\epsilon$, where $\partial F_{\epsilon}$ is the set of edges beginning in $F_{\epsilon}$ and ending outside of $F_{\epsilon}$. Regular trees are examples of non-amenable graphs.

Let $G$ be a group generated by a finite set $S$ which is symmetric (i.e., $S=S^{-1}$ ) and let $T$ be a locally finite rooted tree such that $G$ acts faithfully on $T$. The Schreier graph of this action is defined as an undirected graph with the boundary $\partial T$ as a set of vertices and the set $\partial T \times S$ as a set of edges, where an edge $(u, s) \in \partial T \times S$ connects the vertex $u$ with the vertex $u^{s}$.

Proposition 2 ([21], Proposition 2.2) Let $G$ be a finitely generated subgroup of the group Aut $\left(X^{*}\right)$ and let $T \subseteq X^{*}$ be a $G$-invariant rooted subtree on which $G$ acts level transitively. If all elements of $G$ are almost finitary on $T$, then all components of every Schreier graph of the action of $G$ on $T$ are amenable.

Based on the above results, we can prove Proposition 1 from the introduction.

Proposition 1 Let $X=\left(X_{i}\right)_{i \geq 1}$ be an arbitrary changing alphabet (with a bounded or unbounded degree of vertices) and let $G \leq A u t\left(X^{*}\right)$ be a nearly finitary group. Then $G$ does not contain non-Abelian free subgroups.

Proof Suppose contrary that $F$ is a non-Abelian free subgroup of $G$. Then by Theorem 5, there is $u \in X^{\omega}$ such that one of the following holds:

(i) the group $F$ is contained in the stabilizer of $u$ and for every beginning $w$ of $u$ the group $F$ acts faithfully on the neighborhood $w X_{(|w|+1)}^{\omega}$ of $u$,

(ii) the stabilizer of $u$ in the group $F$ is trivial.

Let $g \in F$ be an arbitrary non-trivial element. Let $U \subseteq X^{\omega}$ and $n \geq 0$ be, respectively, the subset and the number corresponding to $g$ (see Definition 2). Suppose that the case (i) holds. Then $u \notin U$ and, since $U$ is finite, there is a beginning $w$ of $u$ such that $|w| \geq n$ and $w$ is not a beginning of any sequence from $U$. But then the section $g_{\{w\}}$ is trivial, which contradicts the assumption that $F$ acts faithfully on the neighborhood $w X_{(|w|+1)}^{\omega}$. Thus, case (ii) must hold. In this case, the set $T=$ $\left\{w^{g}: w \in X^{*}, w \leq u, g \in F\right\}$ is an $F$-invariant rooted subtree of $X^{*}$ on which $F$ acts level transitively. For every non-trivial $g \in F$, we have $u^{g} \neq u$ and $u^{g} \in \partial T$. Thus, the boundary $\partial T$ is infinite and the sequence $\left(\left|L_{i}\right|\right)_{i \geq 1}$ is divergent, where 
$L_{i}=T \cap X^{i}$. Further, the sequence $\left(\theta_{g, T}(i)\right)_{i \geq 1}$ is bounded, as for all $i \geq n$, we have $\theta_{g, T}(i) \leq \theta_{g, X^{*}}(i) \leq|U|$. Thus, the automorphism $g$ is almost finitary on $T$. Since $g \in F$ was chosen arbitrarily, we have by Proposition 2 that all components of the Schreier graph of the action of $F$ on $T$ are amenable. But the stabilizer of $u$ in the group $F$ is trivial and the component containing $u$ is a regular rooted tree, which is a non-amenable graph. This contradiction finishes the proof of Proposition 1.

\section{Time-varying automata and groups generated by automata}

The combinatorial language of Mealy automata became fruitful in the study of groups acting on regular rooted trees, and it provides various examples of groups having rare and extraordinary geometric, combinatorial and algebraic properties. For more about Mealy automata, groups generated by them and for many problems around this topic see the survey paper [14]. The concept of defining groups by time-varying automata over a changing alphabet is quite new, and it constitutes a natural extension to groups acting on arbitrary (not necessarily regular) spherically homogeneous rooted trees. Note that previously the notion of a time-varying automaton was used only to study structural properties of transducers and concerned automata with the changing transition and output functions but having a fixed alphabet (see [19]). For some explicit and naturally defined representations of groups by time-varying automata and their properties see [27-30,32]. See also [9] where this concept is used to study growth function and Fölner function of groups, as well as the previously mentioned results from [10] .

In the present paper, we refer to time-varying automata over a changing alphabet $X=\left(X_{i}\right)_{i \geq 1}$ as particular finite subsets of the group $\operatorname{Aut}\left(X^{*}\right)$.

Definition 5 A time-varying automaton A over a changing alphabet $X$ is a nonempty finite set $Q \subseteq \operatorname{Aut}\left(X^{*}\right)$ (set of states) together with the automorphisms $q_{i} \in$ $\operatorname{Aut}\left(X_{(i)}^{*}\right)(q \in Q, i \geq 1)$ such that the following two conditions are satisfied:

(i) $q_{1}=q$ for every $q \in Q$,

(ii) for every $q \in Q, i \geq 1$ and $x \in X_{i}$ there is a unique state $q^{\prime} \in Q$ (denoted further by $\varphi_{i}(q, x)$ ) such that the section of the automorphism $q_{i}$ at the letter $x$ is equal to $q_{i+1}^{\prime}$ :

$$
\left(q_{i}\right)_{\{x\}}=q_{i+1}^{\prime} .
$$

The automorphism $q_{i}$ is called the $i$-th transition of the state $q$. For a given $i \geq 1$ the set $Q_{i}=\left\{q_{i}: q \in Q\right\}$ together with the automorphisms $q_{j} \in \operatorname{Aut}\left(X_{(j)}^{*}\right)$ for $j \geq i$ forms a time-varying automaton $A_{i}$ over the changing alphabet $X_{(i)}=\left(X_{j}\right)_{j \geq i}$, which is called the $i$-th transition of the automaton $A$. If the changing alphabet $X$ is fixed (i.e., $X=X_{1}$ by our identification) and for every $i \geq 1$ and every state $q \in Q$ we have $q_{i}=q$, then the automaton $A$ is called a Mealy automaton over the alphabet $X$ (in this case we have: $\varphi_{i}(q, x)=\varphi_{1}(q, x)$ for all $q \in Q, i \geq 1$ and $x \in X$ and to simplify we denote $\left.\varphi(q, x):=\varphi_{1}(q, x)\right)$. We call a state $q \in \operatorname{Aut}\left(X^{*}\right)$ of a time-varying automaton $A$ a neutral state if $q$ is the identity automorphism. 
Fig. 1 The time-varying automaton $\mathcal{A}$ from the introduction

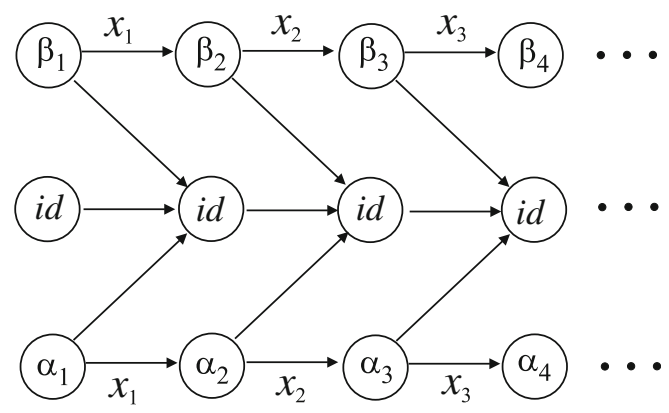

Fig. $2 \ldots$ and the corresponding Mealy automaton (the regular case)

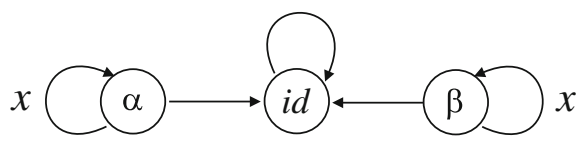

Another convenient way to define a time-varying automaton $A$ is via its diagram, which is a labeled, directed, locally finite graph with the set

$$
\{(i, q): i \geq 1, q \in Q\}
$$

of vertices. Two vertices are connected with an arrow if and only if they are of the form $(i, q)$ and $\left(i+1, \varphi_{i}(q, x)\right)$ for some $i \geq 1, q \in Q$ and $x \in X_{i}$. This arrow is labeled by $x$, starts from the vertex $(i, q)$ and goes to the vertex $\left(i+1, \varphi_{i}(q, x)\right)$. Each vertex $(i, q)$ is labeled by the permutation

$$
\sigma_{i, q}=q_{i}(\epsilon)
$$

which is called the labeling of the state $q$ in the $i$-th transition of $A$. To make the graph of the automaton clear, we will substitute a large number of arrows connecting two given vertices and having the same direction by a one multi-arrow labeled by suitable letters and if the labeling of such a multi-arrow is obvious we will omit this labeling. In the case of a Mealy automaton $A$ over the alphabet $X$, we obtain the Moore diagram of $A$ in which the set of vertices coincides with the set of states and we connect two vertices with an arrow if and only if they are of the form $q$ and $\varphi(q, x)$ for some $q \in Q$ and $x \in X$. Such an arrow is labeled by $x$, starts from the vertex $q$ and goes to the vertex $\varphi(q, x)$. Each vertex $q$ is labeled by its labeling $\sigma_{q}=q_{1}(\epsilon)$. The diagram of the time-varying automaton $\mathcal{A}$ from the introduction is depicted in Fig. 1 . In the regular case, the Moore diagram of the corresponding Mealy automaton presents Fig. 2.

The above notions arise in a natural way by the mechanical interpretation of a timevarying automaton $A$, where $A$ is considered as a machine which being at a moment $i \geq 1$ in a state $q \in Q$ and reading from the input tape a letter $x \in X_{i}$ goes to the state $\varphi_{i}(q, x)$, types on the output tape the letter $x^{\sigma_{i, q}}$, moves both tapes to the next position and then proceeds further to the next moment $i+1$. This interpretation suggests for every word $w=x_{1} x_{2} \ldots x_{t} \in X^{*}$ and $q \in Q$ a very convenient way of computing the image $w^{q}$ from the diagram of $A$. One must find a directed path starting in the 
vertex $(1, q)$ and having the consecutive labels $x_{1}, x_{2}, \ldots, x_{t}$. Such a path exists and is unique. If $\pi_{1}, \pi_{2}, \ldots, \pi_{t}$ are labelings of consecutive vertices in this path, then $x_{i}^{\pi_{i}}$ is the $i$-th letter $(1 \leq i \leq t)$ of the image $w^{q}$.

Definition 6 Let $A$ be a time-varying automaton over a changing alphabet $X$ and let $Q \subseteq A u t\left(X^{*}\right)$ be the set of states. The group generated by the set $Q$ is denoted by $G(A)$ and is called the group generated by the automaton $A$ :

$$
G(A)=\langle q: q \in Q\rangle
$$

Definition 7 Let $X$ be a changing alphabet and let $G \leq A u t\left(X^{*}\right)$ be an arbitrary group. We call $G$ an automaton group (over $X$ ) if $G=G(A)$ for some time-varying automaton $A$ over $X$. If there is an automaton $A$ over the changing alphabet $X$ such that $G$ is topologically generated by the set of states of $A$, then we say that $G$ is topologically generated by the automaton $A$.

Let $A$ be a time-varying automaton over a changing alphabet $X=\left(X_{i}\right)_{i \geq 1}$ in which $X_{i}=\left\{x_{i, 1}, x_{i, 2}, \ldots, x_{i, m_{i}}\right\}$ for every $i \geq 1$. For a given $i \geq 1$, we can write the wreath recursions of the generators $q_{i}(q \in Q)$ of the group $G\left(A_{i}\right)$ generated by the $i$-th transition of the automaton $A$ as follows:

$$
q_{i}=\left(\varphi_{i}\left(q, x_{i, 1}\right)_{i+1}, \varphi_{i}\left(q, x_{i, 2}\right)_{i+1}, \ldots, \varphi_{i}\left(q, x_{i, m_{i}}\right)_{i+1}\right) q_{i}(\epsilon)
$$

The above wreath recursions induce an embedding of the group $G\left(A_{i}\right)$ into the permutational wreath product $G\left(A_{i+1}\right)$ ? $\operatorname{Sym}\left(X_{i}\right)$.

Definition 8 A time-varying automaton $A$ is called self-replicating if for every $i \geq 1$ and every $1 \leq r \leq m_{i}$ we have

$$
\Phi_{i, r}\left(S t_{G\left(A_{i}\right)}(1)\right)=G\left(A_{i+1}\right)
$$

where $\Phi_{i, r}$ is the canonical projection defined in Sect. 2.

Definition 9 Let $\left(K_{i}\right)_{i \geq 1}$ be a sequence of non-trivial subgroups $K_{i} \leq G\left(A_{i}\right)$. We call an automaton $A$ a branching automaton over $\left(K_{i}\right)_{i \geq 1}$ if for every $i \geq 1$ the preimage

$$
\Psi_{i}^{-1}\left(K_{i+1}^{m_{i}}\right)=\Psi_{i}^{-1}(\underbrace{K_{i+1} \times \ldots \times K_{i+1}}_{m_{i}})
$$

is a subgroup of $K_{i}$, where $\Psi_{i}$ is a homomorphism defined in Sect. 2.

Let

$$
Q=\left\{s_{1}, s_{2}, \ldots, s_{k}\right\}
$$

be the set of states in the automaton $A$. The group $G(A)$ can be uniquely determined by the following system of wreath recursions of the automorphisms $\left(s_{j}\right)_{i} \in$ $\operatorname{Aut}\left(X_{(i)}^{*}\right)(1 \leq j \leq k, i \geq 1)$ : 


$$
\left\{\begin{array}{rl}
\left(s_{1}\right)_{i}= & \left(\left(q_{i, 1,1}\right)_{i+1},\left(q_{i, 1,2}\right)_{i+1}, \ldots,\left(q_{i, 1, m_{i}}\right)_{i+1}\right) \pi_{i, 1}, \\
\left(s_{2}\right)_{i}= & \left(\left(q_{i, 2,1}\right)_{i+1},\left(q_{i, 2,2}\right)_{i+1}, \ldots,\left(q_{i, 2, m_{i}}\right)_{i+1}\right) \pi_{i, 2}, \\
\vdots & \vdots \\
\left(s_{k}\right)_{i}= & \left(\left(q_{i, k, 1}\right)_{i+1},\left(q_{i, k, 2}\right)_{i+1}, \ldots,\left(q_{i, k, m_{i}}\right)_{i+1}\right) \pi_{i, k},
\end{array} \quad i \geq 1,\right.
$$

where

$$
q_{i, j, r}=\varphi_{i}\left(s_{j}, x_{i, r}\right), \quad \pi_{i, j}=\left(s_{j}\right)_{i}(\epsilon), \quad 1 \leq j \leq k, \quad 1 \leq r \leq m_{i} .
$$

Conversely, we can consider the set (8) as a set of $k$ symbols. Then an arbitrary system of equations of the form (9), where $\pi_{i, j} \in \operatorname{Sym}\left(X_{i}\right)$ and $q_{i, j, r} \in Q$ for all $i \geq 1,1 \leq j \leq k, 1 \leq r \leq m_{i}$, uniquely defines automorphisms $\left(s_{j}\right)_{i} \in \operatorname{Aut}\left(X_{(i)}^{*}\right)$ in the following recursive way:

$$
\epsilon^{\left(s_{j}\right)_{i}}=\epsilon, \quad i \geq 1, \quad 1 \leq j \leq k,
$$

and if $w=x v \in X_{(i)}^{*}$, where $x=x_{i, r} \in X_{i}$ for some $1 \leq r \leq m_{i}$ and $v \in X_{(i+1)}^{*}$, then

$$
w^{\left(s_{j}\right)_{i}}=x^{\pi_{i, j}} v^{\left(q_{i, j, r}\right)_{i+1}} .
$$

Note that if we identify the symbols $s_{j}(1 \leq j \leq k)$ from the set $Q$ with the automorphisms $\left(s_{j}\right)_{1} \in \operatorname{Aut}\left(X^{*}\right)$, then we obtain a time-varying automaton over the changing alphabet $X$ and with the set $Q_{1}=\left\{\left(s_{j}\right)_{1}: 1 \leq j \leq k\right\}$ as a set of states. If the automaton $A$ is a Mealy automaton, then the system of wreath recursions defining the group $G(A)$ consists of a finite number of equations:

$$
\left\{\begin{array}{l}
s_{1}=\left(q_{11}, q_{12}, \ldots, q_{1 m}\right) \pi_{1}, \\
s_{2}=\left(q_{21}, q_{22}, \ldots, q_{2 m}\right) \pi_{2}, \\
\vdots \\
s_{k}=\left(q_{k 1}, q_{k 2}, \ldots, q_{k m}\right) \pi_{k}
\end{array}\right.
$$

where

$$
q_{j r}=\varphi\left(s_{j}, x_{1, r}\right), \quad \pi_{j}=s_{j}(\epsilon), \quad 1 \leq j \leq k, \quad 1 \leq r \leq m_{1} .
$$

\section{Disappearing group words of an automaton}

Let $A$ be an arbitrary time-varying automaton over a changing alphabet $X=\left(X_{i}\right)_{i \geq 1}$ in which $X_{i}=\left\{x_{i, 1}, x_{i, 2}, \ldots, x_{i, m_{i}}\right\}$ for every $i \geq 1$. We can define the automaton $A$ by a $(k+1)$-element $(k \geq 1)$ set of symbols

$$
Q=\left\{s_{1}, \ldots, s_{k}\right\} \cup\{i d\}
$$


representing the states of $A$ with the symbol $i d$ representing a neutral state together with the system of equations:

$$
\left\{\begin{aligned}
\left(s_{1}\right)_{i}= & \left(\left(q_{i, 1,1}\right)_{i+1},\left(q_{i, 1,2}\right)_{i+1}, \ldots,\left(q_{i, 1, m_{i}}\right)_{i+1}\right) \pi_{i, 1}, \\
\left(s_{2}\right)_{i}= & \left(\left(q_{i, 2,1}\right)_{i+1},\left(q_{i, 2,2}\right)_{i+1}, \ldots,\left(q_{i, 2, m_{i}}\right)_{i+1}\right) \pi_{i, 2}, \quad \\
\vdots & \vdots \\
\left(s_{k}\right)_{i}= & \left(\left(q_{i, k, 1}\right)_{i+1},\left(q_{i, k, 2}\right)_{i+1}, \ldots,\left(q_{i, k, m_{i}}\right)_{i+1}\right) \pi_{i, k},
\end{aligned}\right.
$$

where $q_{i, j, r} \in Q$ and $\pi_{i, j} \in \operatorname{Sym}\left(X_{i}\right)$ for $i \geq 1,1 \leq j \leq k, 1 \leq r \leq m_{i}$. The equations (11) are wreath recursions of the corresponding automorphisms $\left(s_{j}\right)_{i} \in$ $\operatorname{Aut}\left(X_{(i)}^{*}\right)$ for $i \geq 1$ and $1 \leq j \leq k$ (in (11), we omit the wreath recursion $i d_{i}=$ $\left(i d_{i+1}, \ldots, i d_{i+1}\right)$ of the identity automorphism $i d_{i} \in \operatorname{Aut}\left(X_{(i)}^{*}\right)$ corresponding to the symbol $i d$ ).

For each $i \geq 1$, we deal with the generating set

$$
Q_{i}=\left\{\left(s_{1}\right)_{i}, \ldots,\left(s_{k}\right)_{i}\right\} \cup\left\{i d_{i}\right\}
$$

of the group $G\left(A_{i}\right)$ generated by the $i$-th transition of the automaton $A$ as a basis of a free group $F=F\left(Q_{i}\right)$ of rank $k+1$. We call an arbitrary group word $W \in F\left(Q_{i}\right)$ reduced if it does not contain the trivial subwords, i.e., the subwords of the form:

$$
i d_{i}, \quad i d_{i}^{-1}, \quad\left(s_{j}\right)_{i}\left(s_{j}\right)_{i}^{-1}, \quad\left(s_{j}\right)_{i}^{-1}\left(s_{j}\right)_{i} \quad 1 \leq j \leq k
$$

Otherwise we call $W$ reducible. If $W$ is reducible, then by consecutive deletion of trivial subwords, we can obtain a unique reduced group word, which we call a reduced form of $W$ and denote it by $\bar{W}$. By using the system (11), for every letter $x=x_{i, r} \in$ $X_{i}\left(1 \leq r \leq m_{i}\right)$, we can define in the recursive way the section $W_{\{x\}}$ of the group word $W$ at $x$. Namely, if $W=\varepsilon$, the empty group word, or $W \in\left\{i d_{i}, i d_{i}^{-1}\right\}$, then $W_{\{x\}}=\varepsilon$; if $W=\left(s_{j}\right)_{i}$, or $W=\left(s_{j}\right)_{i}^{-1}$ for some $1 \leq j \leq k$, then

$$
W_{\{x\}}=\left(q_{i, j, r}\right)_{i+1}, \quad \text { or } \quad W_{\{x\}}=\left(q_{i, j, r^{\prime}}\right)_{i+1}^{-1},
$$

respectively, where $r^{\prime} \in\left\{1,2, \ldots, m_{i}\right\}$ is the unique element such that $x_{i, r^{\prime}}=x_{i, r}^{\pi_{i, j}^{-1}}$; if $W=U V$, where $V \in F\left(Q_{i}\right)$ and $U \in\left\{i d_{i}, i d_{i}^{-1},\left(s_{j}\right)_{i},\left(s_{j}\right)_{i}^{-1}\right\}$ for some $1 \leq j \leq k$, then

$$
W_{\{x\}}=\overline{U_{\{x\}} V_{\left\{x^{\prime}\right\}}},
$$

where

$$
x^{\prime}= \begin{cases}x, & U \in\left\{i d_{i}, i d_{i}^{-1}\right\} \\ x^{\pi_{i, j}}, & U=\left(s_{j}\right)_{i} \\ x^{\pi_{i, j}^{-1}}, & U=\left(s_{j}\right)_{i}^{-1}\end{cases}
$$


Finally, we define recursively the section $W_{\{w\}}$ of $W$ at any vertex $w \in X_{(i)}^{*}: W_{\epsilon}=\bar{W}$, and if $w=v x$ for some $v \in X_{(i)}^{*}$ and $x \in X_{|v|+i}$, then $W_{\{w\}}=\left(W_{\{v\}}\right)_{\{x\}}$. In particular, we see that the section $W_{\{w\}}$ is a reduced group word over the set $Q_{i+|w|}$, i.e., $W_{\{w\}} \in F\left(Q_{i+|w|}\right)$ and if $W$ defines an element $g \in G\left(A_{i}\right)$, then the section $W_{\{w\}}$ defines the element $g_{\{w\}} \in G\left(A_{i+|w|}\right)$.

Remark 2 Further, we will use the following notation. If $W \in F\left(Q_{i}\right)$ for some $i \geq 1$ and $g \in G\left(A_{i}\right)$ is the element defined by $W$, then for every word $w \in X_{(i)}^{*}$, we will write $w^{W}$ for the image $w^{g}$; by $W(\epsilon)$, we will denote the root permutation of the automorphism $g$.

Since the group words (13) define the identity automorphism, we obtain by the above construction as well as in view of the formulas (6)-(7) the following properties of the section $W_{\{w\}}$ :

$-W_{\{w\}}=(\bar{W})_{\{w\}}$,

- $\left(W W^{\prime}\right)_{\{w\}}=\overline{W_{\{w\}} W_{\left\{w^{\prime}\right\}}^{\prime}}$, where $w^{\prime}=w^{W}$,

$-\left(W^{-1}\right)_{\{w\}}=\left(W_{\left\{w^{\prime \prime}\right\}}\right)^{-1}$, where $w^{\prime \prime}=w^{W^{-1}}$,

- if $v$ is a prefix of $w$, then $\left|W_{\{w\}}\right| \leq\left|W_{\{v\}}\right|$

for all $i \geq 1, W, W^{\prime} \in F\left(Q_{i}\right)$ and $w, v \in X_{(i)}^{*}$.

Definition 10 A group word $W \in F\left(Q_{1}\right)$ defining the neutral element in the group $G\left(A_{1}\right)$ is called a disappearing group word of the automaton $A$ if there is a number $n_{0}$ such that $W_{\{w\}}=\varepsilon$ for every word $w \in X^{n_{0}}$. We call the smallest number $n_{0}$ with the above property the depth of $W$ and denote it by $\lambda(W)$.

Obviously, not every group word defining the neutral element must be disappearing. However, in the next section, we introduce the class of time-varying automata $A$ such that every group word $W \in F\left(Q_{1}\right)$ defining the neutral element in $G\left(A_{1}\right)$ is also a disappearing group word (see Proposition 5).

Directly by the above properties of the section $W_{\{w\}}$, we see that if group words $W, V \in F\left(Q_{1}\right)$ are disappearing, then the following group words:

$$
W V, \quad W^{-1}, \quad U^{-1} W U
$$

are also disappearing, where $U \in F\left(Q_{1}\right)$ is arbitrary. Moreover, for their depths, we have:

$-\lambda(W)=\lambda(\bar{W})$,

$-\lambda(W V) \leq \max \{\lambda(W), \lambda(V)\}$

$-\lambda\left(W^{-1}\right)=\lambda(W)$,

$-\lambda\left(U^{-1} W U\right) \leq \lambda(W)$.

Definition 11 If for every integer $n$, there is a disappearing group word $W$ of the automaton $A$ such that $\lambda(W) \geq n$, then we say that $A$ has disappearing group words of unbounded depth. 
Proposition 3 Suppose that the automaton A has disappearing group words of unbounded depth and every group word $W \in F\left(Q_{1}\right)$ which defines the neutral element in $G\left(A_{1}\right)$ is disappearing. Then the group generated by this automaton is not finitely presented.

Proof Suppose that the group $G(A)=G\left(A_{1}\right)$ is finitely presented. Then there is a finite subset $R \subseteq F\left(Q_{1}\right)$ of group words defining the neutral element in $G\left(A_{1}\right)$ such that every reduced group word $W \in F\left(Q_{1}\right)$ which defines the neutral element in $G\left(A_{1}\right)$ is freely equal to a product of conjugations of some group words from $R$ or their inverses. Since every group word from $R$ is disappearing, we have $\lambda(W) \leq$ $\max \{\lambda(V): V \in R\}$, which contradicts with the assumption that the automaton $A$ has disappearing group words of unbounded depth.

\section{Automata with the nucleus}

In the sequel, we will use the following notation. If $W \in F(Q)$ is an arbitrary group word over the set (10), then for every $i \geq 1$, we denote by $W_{(i)} \in F\left(Q_{i}\right)$ a group word over the set (12) which arises from $W$ by substitution of every occurrence of $i d$ by $i d_{i}$, every occurrence of $i d^{-1}$ by $i d_{i}^{-1}$, and for each $1 \leq j \leq k$ every occurrence of $s_{j}$ by $\left(s_{j}\right)_{i}$ and every occurrence of $s_{j}^{-1}$ by $\left(s_{j}\right)_{i}^{-1}$. We call the group word $W_{(i)}$ the $i$-th transition of $W$. Similarly, for any subset $S \subseteq F(Q)$ and every $i \geq 1$, we denote:

$$
S_{(i)}=\left\{W_{(i)}: W \in S\right\} \subseteq F\left(Q_{i}\right) .
$$

Let $A$ be a time-varying automaton defined by (10)-(11).

Definition 12 We call the automaton $A$ an automaton with a nucleus if there is a finite subset $S \subseteq F(Q)$ (further called a nucleus) of non-empty reduced group words which satisfy the following conditions:

(i) for every $V \in F\left(Q_{1}\right)$ there is an integer $n_{0}$ such that for every $w \in X^{n_{0}}$ we have $V_{\{w\}} \in S_{\left(n_{0}+1\right)} \cup\{\varepsilon\}$

(ii) for every $W \in S$ there is an infinite sequence $x_{1} x_{2} \ldots \in X^{\omega}$ such that for every $i \geq 1$ we have:

$$
\left(W_{(i)}\right)_{\left\{x_{i}\right\}}=W_{(i+1)}, \quad\left(W_{(i)}\right)_{\{x\}}=\varepsilon \quad \text { for } \quad x \in X_{i} \backslash\left\{x_{i}\right\} .
$$

If, additionally, for every $W \in S$ and every $i \geq 1$ the following inequality holds:

(ii') $x_{i}^{W_{(i)}} \neq x_{i}$,

then the automaton $A$ is called an automaton with a path-active nucleus and the set $S$ is called a path-active nucleus of $A$.

Proposition 4 If the automaton $A$ is an automaton with a path-active nucleus, then the group $G(A)=G\left(A_{1}\right)$ is a nearly finitary group. 
Proof Suppose that the automaton $A$ is an automaton with a path-active nucleus and let $S$ be a path-active nucleus of $A$. Let $g \in G\left(A_{1}\right)$ be arbitrary and let $V \in F\left(Q_{1}\right)$ be a group word defining $g$. Let $n_{0}$ be an integer such that for every $w \in X^{n_{0}}$ we have $V_{\{w\}} \in S_{\left(n_{0}+1\right)} \cup\{\varepsilon\}$. Let $\mathcal{U}_{0}$ be the subset of $X^{n_{0}}$ which consists of all words $w$ such that the corresponding section $V_{\{w\}}$ is not empty, i.e., $V_{\{w\}} \in S_{\left(n_{0}+1\right)}$. If $\mathcal{U}_{0}$ is empty, then obviously the element $g$ is nearly finitary. If $\mathcal{U}_{0}$ is non-empty, then we can write $\mathcal{U}_{0}=\left\{w_{1}, \ldots, w_{t}\right\}$ and for every $1 \leq l \leq t$ there is $W_{l} \in S$ such that

$$
V_{\left\{w_{l}\right\}}=\left(W_{l}\right)_{\left(n_{0}+1\right)} .
$$

Let $y_{l, 1} y_{l, 2} \ldots \in X^{\omega}(1 \leq l \leq t)$ be an infinite sequence corresponding to $W_{l}$. Let us consider the subset $\mathcal{U}=\left\{u_{1}, \ldots, u_{t}\right\} \subseteq X^{\omega}$, where:

$$
u_{l}=w_{l} y_{l, n_{0}+1} y_{l, n_{0}+2} \ldots, \quad 1 \leq l \leq t
$$

Then for each $1 \leq l \leq t$, we have $u_{l}^{g}=w_{l}^{g} y_{l, n_{0}+1}^{\prime} y_{l, n_{0}+2}^{\prime} \ldots$, where

$$
y_{l, n_{0}+i}^{\prime}=y_{l, n_{0}+i}^{\left(W_{l}\right)_{\left(n_{0}+i\right)}}, \quad i \geq 1 .
$$

Since the set $S$ is a path-active nucleus, we obtain $y_{l, n_{0}+i}^{\prime} \neq y_{l, n_{0}+i}$. Hence, $u^{g} \neq u$ for every $u \in \mathcal{U}$. Next, let $w \in X^{*}$ and $|w| \geq n_{0}$. If neither of the words $w_{l}$ for $1 \leq l \leq t$ is a prefix of $w$, then we see by the definition of the set $\mathcal{U}_{0}$ that the section $V_{\{w\}}$ is empty. If there is $1 \leq l \leq t$ such that $w_{l}$ is a prefix of $w$ but $w$ is not a beginning of the sequence $u_{l}$, then by Definition 12, (ii) we see that the section $V_{\{w\}}$ is empty. Consequently, for any word $w \in X^{*}$ with the length $|w|>n_{0}$ and such that $w$ is not a beginning of any sequence from $\mathcal{U}$, the label $g(w)$ is trivial. Thus, the element $g$ is nearly finitary.

Proposition 5 If the automaton $A$ is an automaton with a path-active nucleus, then every group word $V \in F\left(Q_{1}\right)$ which defines the neutral element in $G\left(A_{1}\right)$ is a disappearing group word.

Proof Suppose it is not true, and let $V \in F\left(Q_{1}\right)$ be a group word defining the neutral element which is not a disappearing group word. Let $n_{0}$ be an integer such that $V_{\{w\}} \in$ $S_{\left(n_{0}+1\right)} \cup\{\varepsilon\}$ for every $w \in X^{n_{0}}$, where $S$ is a path-active nucleus of the automaton $A$. Since $V$ is not disappearing, there is $w_{0} \in X^{n_{0}}$ such that $V_{\left\{w_{0}\right\}}=W_{\left(n_{0}+1\right)}$ for some $W \in S$. Since the set $S$ is a path-active nucleus, there is a letter $x \in X_{n_{0}+1}$ such that $x^{W_{\left(n_{0}+1\right)}} \neq x$. But then $x^{V_{\left\{w_{0}\right\}}}=x^{W_{\left(n_{0}+1\right)}} \neq x$, which is impossible as the section $V_{\left\{w_{0}\right\}}$ defines the neutral element in the group $G\left(A_{n_{0}+1}\right)$.

Corollary 3 If the automaton $A$ is an automaton with a path-active nucleus, then the group $G(A)$ does not contain non-Abelian free subgroups. Additionally, if A has disappearing group words of unbounded depth, then the group $G(A)$ is not finitely presented. 


\section{The proof of Theorem 1}

Let $(H, X)$ be an arbitrary non-Abelian finite simple transitive permutation group on a set $X$. At first, we show that the group $H$ has a hooked triple. Let us start with the following

Proposition 6 The group $H$ has a 2-element generating set $\{\alpha, \beta\}$ such that $\alpha$ is an involution and $\beta$ is not an $m$-cycle, where $m=|X|$.

Proof (of Proposition 6) By the main result in [24], there is a conjugacy class $C$ of $H$ such that for every non-trivial element $\alpha \in H$, there is an element $\beta \in C$ such that $H$ is generated by the set $\{\alpha, \beta\}$. We distinguish two cases.

Case 1: $m$ is even. Let $\alpha \in H$ be any involution (the existence of such an element follows from the famous Feit-Thompson theorem). By the above, we can choose $\beta \in C$ such that the elements $\alpha, \beta$ generate $H$. Since the group $H$ is perfect, the permutation $\beta$ is even, and hence, it cannot be an $m$-cycle. Thus, the set $\{\alpha, \beta\}$ satisfies the claim in this case.

Case 2: $m$ is odd. In this case, we use the following

Lemma 1 If the group $H$ contains an $m$-cycle, then $H$ is primitive.

Proof (of Lemma 1) Suppose contrarily that $H$ is imprimitive and it contains an $m$-cycle. Let $\mathcal{B}$ be any non-trivial block system of $H$. Let $H \rightarrow \operatorname{Sym}(\mathcal{B}), h \mapsto L_{h}$ be the induced action of $H$ on $\mathcal{B}$. If $h_{0} \in H$ is an $m$-cycle, then the permutation $L_{h_{0}} \in \operatorname{Sym}(\mathcal{B})$ is an $n$-cycle, where $n=|\mathcal{B}|<m$. Thus, the $n$-th power of $h_{0}$ is a non-trivial element from the kernel of this action, which is impossible as $H$ is simple. This finishes the proof of Lemma 1.

Further, we can assume that the group $H$ contains an $m$-cycle (otherwise we can choose a suitable generating set directly basing on the Stein result). By Lemma 1, we can use the following classification due to Feit and Jones [11,17] (below, we use the standard notation for the general affine group of degree one $A G L_{1}(q)$, the alternating group $A_{n}$ and the symmetric group $S_{n}$, the projective general linear group $P G L_{k}(q)$, the projective semilinear group $P \Gamma L_{k}(q)$, the projective special linear group $P S L_{k}(q)$, and the Mathieu groups $\left.M_{n}\right)$ :

Theorem 6 ([17], Theorem 3) Let $G$ be a primitive permutation group of degree $m$ which contains an m-cycle. Then one of the following holds.

- $G \leq A G L_{1}(m), m$ is a prime; or

- $G=A_{m}$ or $S_{m}$; or

- $P G L_{k}(q) \leq G \leq P \Gamma L_{k}(q)$, where $k \geq 2, q$ a prime power, $G$ acting naturally on the projective space with $m=\left(q^{k}-1\right) /(q-1)$ points; or

- $m=11, G=P S L_{2}(11)$ or $M_{11}$; or

$-m=23, G=M_{23}$.

Note that if $m$ is a prime, then the group $A G L_{1}(m)$ contains a unique cyclic group of order $m$ and this cyclic group is normal in $A G L_{1}(m)$. Further, the group $P G L_{k}(q)$ is a 
normal subgroup of $P \Gamma L_{k}(q)$ and $P G L_{k}(q)$ is simple if and only if the numbers $k, q-$ 1 are coprime and $(k, q) \neq(2,2)$, which then implies $P G L_{k}(q)=P S L_{k}(q)$. Hence, since $H$ is simple, we obtain by the above classification the following possibilities for the group $H$ :

- $H=A_{m} ;$ or

- $H=P S L_{k}(q)$ for some $k \geq 2$ and $q$ a prime power such that the numbers $k$ and $q-1$ are coprime and $m=\left(q^{k}-1\right) /(q-1)$; or

- $m=11, H=P S L_{2}(11)$ or $M_{11}$; or

- $m=23, H=M_{23}$.

By [18], we know that the projective special linear groups $P S L_{2}(q)$ with $q \neq 9$ can be generated by two elements $\alpha, \beta$ such that $\alpha$ is an involution and $\beta$ is of order 3 . By [12], this property also holds for $P S L_{3}(q)$ with $q \neq 4$ and by [7] for alternating groups $A_{n}$ of degree $n \geq 5, n \neq 6,7,8$. For the alternating group $A_{7}$ and for the group $\mathrm{PSL}_{4}(2)=A_{8}$, the element $\beta$ can be chosen to have order 4; by [8], the same holds for the groups $M_{11}, M_{23}$. If $H$ is one of the above groups, then $m \geq 5$, and hence, the element $\beta$ cannot be an $m$-cycle. Further, if $k \geq 4$ and $(k, q) \notin\{(4,2),(11,2)\}$, then by [16] (see Table II, p. 763), the corresponding conjugacy class $C$ in the group $P S L_{k}(q)$ can be chosen in such a way that the order $o(\beta)$ of any element $\beta \in C$ satisfies the following divisibility:

$$
\begin{array}{ll}
o(\beta) \mid\left(q^{l+2}-1\right)\left(q^{l-2}-1\right) & \text { if } \quad k=2 l, \quad 2 \nmid l, \quad l \geq 2, \\
o(\beta) \mid\left(q^{l+1}-1\right)\left(q^{l-1}-1\right) & \text { if } \quad k=2 l, \quad 2 \mid l, \quad l \geq 2, \\
o(\beta) \mid\left(q^{l+1}-1\right)\left(q^{l}-1\right) & \text { if } \quad k=2 l+1, \quad l \geq 2 .
\end{array}
$$

In particular, $\beta$ cannot be an $m$-cycle, as in each case, one can easily check that the number $m=\left(q^{k}-1\right) /(q-1)$ does not divide the right-hand side of the above divisibility. Finally, if $H=P S L_{11}(2)=S L_{11}$ (2), then by [25], we see that $H$ is generated by an involution and an element of order 11 . Since in this case, we have $m=2^{11}-1>11$, the element of order 11 cannot be an $m$-cycle. This finishes the proof of Proposition 6.

Proposition 7 The group $H$ has a hooked triple.

Proof (of Proposition 7) Let $\{\alpha, \beta\}$ be the generating set from Proposition 6 and let $\alpha=\tau_{1} \cdot \ldots \cdot \tau_{s}, \beta=\sigma_{1} \cdot \ldots \cdot \sigma_{t}$ be decompositions of $\alpha, \beta$ into the products of nontrivial disjoint cycles. Since each $\tau_{j}(1 \leq j \leq s)$ is a transposition, its support consists of two elements. Thus, if the claim were false, for each $1 \leq j \leq s$, the support of the transposition $\tau_{j}$ would be either contained in the set of fixed points of $\beta$, or it would be contained in the support of a cycle $\sigma_{j^{\prime}}$ for some $1 \leq j^{\prime} \leq t$. But then the group generated by $\alpha, \beta$ would not be transitive, which finishes the proof of Proposition 7.

Now we can prove Theorem 1 from the introduction.

Theorem 1 The inverse limit $W_{\infty}=\ldots 2 \mathrm{H}_{2}$ 2 $\mathrm{H}_{1}$ is topologically generated by the time-varying automaton $\mathcal{A}$ defined by (2)-(3). 
Proof For any $i, j \geq 1$, we denote by $a_{i, j}, b_{i, j} \in A u t\left(X_{(i)}^{*}\right)$ the automorphisms which arise from the $i$-th transitions $a_{i}, b_{i} \in A u t\left(X_{(i)}^{*}\right)$ of the states $a, b$ by the substitution of all labels $a_{i}(w), b_{i}(w)$ at words $w \in X_{(i)}^{*}$ with $|w| \geq j$ by the trivial permutations. Also, let us denote by $W_{i, j}$ the subgroup of all automorphisms $g \in \operatorname{Aut}\left(X_{(i)}^{*}\right)$ which satisfy for every word $w \in X_{(i)}^{*}$ the following condition: If $|w|<j$, then $g(w) \in$ $H_{i+|w|}$, and if $|w| \geq j$, then $g(w)=i d$. We see that $W_{i, j}$ is isomorphic to the $j$-iterated wreath product of the form

$$
H_{i+j-1} 2 \ldots 2 H_{i+1} \prec H_{i} .
$$

It suffices to show that $W_{i, j}=\left\langle a_{i, j}, b_{i, j}\right\rangle$ for all $i, j \geq 1$. This is true for $j=1$ as we have: $a_{i, 1}=\alpha_{i}, b_{i, 1}=\beta_{i}$ and $W_{i, 1}=H_{i}=\left\langle\alpha_{i}, \beta_{i}\right\rangle$. We inductively assume that the statement is true for all $i \geq 1$ and for some fixed $j_{0} \geq 1$, that is we have

$$
W_{i, j_{0}}=\left\langle a_{i, j_{0}}, b_{i, j_{0}}\right\rangle, \quad i \geq 1
$$

Let us choose $i_{0} \geq 1$ arbitrarily and let us denote: $g=a_{i_{0}, j_{0}+1}, h=b_{i_{0}, j_{0}+1}$. We must show that $G=W_{i_{0}, j_{0}+1}$, where $G:=\langle g, h\rangle$. Obviously, we have $G \subseteq W_{i_{0}, j_{0}+1}$. By wreath recursions (3), we have

$$
\left\{\begin{array}{l}
g=\left(g^{\prime}, i d, \ldots, i d\right) \alpha \\
h=\left(h^{\prime}, i d, \ldots, i d\right) \beta
\end{array}\right.
$$

where $g^{\prime}=a_{i_{0}+1, j_{0}}, h^{\prime}=b_{i_{0}+1, j_{0}}, \alpha=\alpha_{i_{0}}, \beta=\beta_{i_{0}}$.

By the definition of the triple $\left(\alpha, \beta, x_{i_{0}}\right)$, we can choose two non-trivial cycles from decompositions of $\alpha$ and $\beta$ into the products of disjoint cycles, the first cycle from the decomposition of $\alpha$ and the second cycle from the decomposition of $\beta$, such that $x_{i_{0}}$ is the only letter which belongs to both these cycles. Let $l_{1}, l_{2}$ be the lengths of these two cycles, respectively. Then by (14), we have:

$$
\left(g^{l_{1}}\right)_{\left\{x_{i_{0}}\right\}}=g^{\prime}, \quad\left(h^{l_{2}}\right)_{\left\{x_{i_{0}}\right\}}=h^{\prime}, \quad g^{l_{1}}(\epsilon)=i d, \quad h^{l_{2}}(\epsilon)=i d,
$$

and also

$$
\left(g^{l_{1}}\right)_{\{x\}}=i d \text { or }\left(h^{l_{2}}\right)_{\{x\}}=i d
$$

for every letter $x \in X_{i_{0}} \backslash\left\{x_{i_{0}}\right\}$.

Let $f \in W_{i_{0}+1, j_{0}}$ be arbitrary. By the inductive assumption, we have

$$
W_{i_{0}+1, j_{0}}=\left\langle g^{\prime}, h^{\prime}\right\rangle \text {. }
$$

Since the group $W_{i_{0}+1, j_{0}}$ is perfect, there is a group word $V=V\left(g^{\prime}, h^{\prime}\right)$ on the letters $g^{\prime}, h^{\prime}$ such that $V$ represents $f$ and the sums of exponents on both $g^{\prime}$ and $h^{\prime}$ are equal to zero. Then by (15)-(16), we see that for the wreath recursion of the element

$$
f^{\prime}=V\left(g^{l_{1}}, h^{l_{2}}\right) \in G
$$


we have: $f^{\prime}=(f, i d, \ldots, i d)$. Since $f$ was chosen arbitrarily, we see that the element with the wreath recursion $\left(g^{\prime}, i d, \ldots, i d\right)$ and the element with the wreath recursion $\left(h^{\prime}, i d, \ldots, i d\right)$ both belong to $G$. Consequently, by (14), we have $H_{i_{0}} \subseteq G$. Next, since the group $H_{i_{0}}$ is transitive, for any letter $x \in X_{i_{0}}$, we can choose an element $\gamma \in H_{i_{0}} \subseteq G$ such that the wreath recursion of the conjugate $\gamma^{-1} f^{\prime} \gamma$ is of the form $(i d, \ldots, i d, f, i d, \ldots, i d)$, where the position of $f$ corresponds to the letter $x$. Consequently, we obtain that the direct power $W_{i_{0}+1, j_{0}}^{m}$, where $m=\left|X_{i_{0}}\right|$, is a subgroup of $G$. Hence, we have

$$
W_{i_{0}, j_{0}+1}=W_{i_{0}+1, j_{0}}^{m} \rtimes H_{i_{0}} \subseteq G,
$$

which finishes the proof of Theorem 1 .

\section{The proof of other properties of the group $G(\mathcal{A})$}

We start with the following

Proposition 8 The automaton $\mathcal{A}$ is an automaton with a path-active nucleus. Namely, the set

$$
S=\left\{a, a^{-1}, b, b^{-1}, a^{-1} b, b^{-1} a\right\}
$$

is a path-active nucleus of the automaton $\mathcal{A}$.

Proof For every $i \geq 1$ let us denote:

$$
y_{i}=x_{i}^{a_{i}}, \quad z_{i}=x_{i}^{b_{i}} .
$$

Since in the decompositions of the permutations $\alpha_{i}, \beta_{i}$ into the products of disjoint cycles, the two cycles which contain the letter $x_{i}$ are non-trivial and $x_{i}$ is the only letter which belongs to both supports of these two cycles, we obtain the following inequalities:

$$
y_{i} \neq x_{i}, \quad z_{i} \neq x_{i}, \quad z_{i} \neq y_{i}
$$

Directly by the defining wreath recursions (3), we obtain for any letter $x \in X_{i}$ :

$$
\begin{aligned}
& \left(a_{i}\right)_{\{x\}}=\left\{\begin{array}{ll}
a_{i+1}, & x=x_{i}, \\
\varepsilon, & \text { otherwise, }
\end{array} \quad\left(a_{i}^{-1}\right)_{\{x\}}= \begin{cases}a_{i+1}^{-1}, & x=y_{i}, \\
\varepsilon, & \text { otherwise },\end{cases} \right. \\
& \left(b_{i}\right)_{\{x\}}=\left\{\begin{array}{ll}
b_{i+1}, & x=x_{i}, \\
\varepsilon, & \text { otherwise, }
\end{array} \quad\left(b_{i}^{-1}\right)_{\{x\}}= \begin{cases}b_{i+1}^{-1}, & x=z_{i}, \\
\varepsilon, & \text { otherwise }\end{cases} \right.
\end{aligned}
$$

In particular, by the inequalities (18), we see that for every integer $N \notin\{-1,0,1\}$ and every $x \in X_{i}$, there are integers $N^{\prime}, N^{\prime \prime}$ with $\left|N^{\prime}\right|<|N|,\left|N^{\prime \prime}\right|<|N|$ and such that $\left(a_{i}^{N}\right)_{\{x\}}=a_{i+1}^{N^{\prime}},\left(b_{i}^{N}\right)_{\{x\}}=b_{i+1}^{N^{\prime \prime}}$. Further, we have for any $x \in X_{i}$ : 


$$
\begin{aligned}
& \left(a_{i}^{-1} b_{i}\right)_{\{x\}}= \begin{cases}a_{i+1}^{-1} b_{i+1}, & x=y_{i}, \\
\varepsilon, & \text { otherwise, }\end{cases} \\
& \left(b_{i}^{-1} a_{i}\right)_{\{x\}}= \begin{cases}b_{i+1}^{-1} a_{i+1}, & x=z_{i}, \\
\varepsilon, & \text { otherwise, }\end{cases}
\end{aligned}
$$

and also:

$$
\begin{aligned}
& \left(a_{i} b_{i}\right)_{\{x\}}=\left\{\begin{array}{l}
a_{i+1}, x=x_{i}, \\
b_{i+1}, x=x_{i}^{a_{i}^{-1}}, \\
\varepsilon, \quad \text { otherwise, }
\end{array} \quad\left(b_{i}^{-1} a_{i}^{-1}\right)_{\{x\}}=\left\{\begin{array}{l}
b_{i+1}^{-1}, x=z_{i}, \\
a_{i+1}^{-1}, x=y_{i}^{b_{i}}, \\
\varepsilon, \quad \text { otherwise, }
\end{array}\right.\right. \\
& \left(a_{i} b_{i}^{-1}\right)_{\{x\}}=\left\{\begin{array}{ll}
a_{i+1}, & x=x_{i}, \\
b_{i+1}^{-1}, & x=z_{i}^{a_{i}^{-1}}, \\
\varepsilon, & \text { otherwise, }
\end{array} \quad\left(b_{i} a_{i}^{-1}\right)_{\{x\}}= \begin{cases}b_{i+1}, & x=x_{i}, \\
a_{i+1}^{-1}, & x=y_{i}^{b_{i}^{-1}}, \\
\varepsilon, & \text { otherwise, }\end{cases} \right. \\
& \left(a_{i}^{-1} b_{i}^{-1}\right)_{\{x\}}=\left\{\begin{array}{ll}
a_{i+1}^{-1}, & x=y_{i}, \\
b_{i+1}^{-1}, & x=z_{i}^{a_{i},} \\
\varepsilon, & \text { otherwise, }
\end{array} \quad\left(b_{i} a_{i}\right)_{\{x\}}=\left\{\begin{array}{l}
b_{i+1}, x=x_{i}, \\
a_{i+1}, x=x_{i}^{b_{i}^{-1}}, \\
\varepsilon, \quad \text { otherwise. }
\end{array}\right.\right.
\end{aligned}
$$

Thus, for every $i \geq 1$ and every reduced group word $W$ over the set

$$
Q_{i}=\left\{a_{i}, b_{i}, i d_{i}\right\}
$$

we obtain: if $|W|=2$, then $\left|W_{\{x\}}\right|<|W|$ for any $x \in X_{i}$, unless $W=a_{i}^{-1} b_{i}$ and $x=y_{i}$, or $W=b_{i}^{-1} a_{i}$ and $x=z_{i}$. But, if $|W| \geq 3$, then $W$ contains a subword of length 2 which is neither of the form $a_{i}^{-1} b_{i}$ nor $b_{i}^{-1} a_{i}$. Thus, the formulas (23)-(25) together with the above observation on sections of the powers $a_{i}^{N}, b_{i}^{N}, N \notin\{-1,0,1\}$ give: if $|W| \geq 3$, then $\left|W_{\{x\}}\right|<|W|$ for every letter $x \in X_{i}$. Consequently, for every group word $W \in F\left(Q_{1}\right)$ there is $n \geq 1$ such that $\left|W_{\{w\}}\right| \leq 2$ for every $w \in X^{n-1}$, which together with the formulas (19)-(25) implies that there is $n^{\prime} \geq n$ such that $W_{\{w\}} \in S_{\left(n^{\prime}\right)} \cup\{\varepsilon\}$ for every $w \in X^{n^{\prime}-1}$. Directly by the formulas (19)-(22), we see that for every group word from the set $S$, we can choose from $X^{\omega}$ one of the following three sequences:

$x_{1} x_{2} x_{3} \ldots, \quad y_{1} y_{2} y_{3} \ldots, \quad z_{1} z_{2} z_{3} \ldots$,

such that the item (ii) from Definition 12 is satisfied for this group word. Thus, the set $S$ is a nucleus of the automaton $\mathcal{A}$. Moreover, by the inequalities (18) and by $y_{i}^{a_{i}^{-1} b_{i}}=z_{i}$, we see that $S$ is a path-active nucleus of $\mathcal{A}$.

As a direct corollary of Propositions 1, 4, 8, we obtain Theorem 2. In spite of this result, we easily obtain the following

Proposition 9 The semigroup generated by the states $a, b$ is free. 
Proof Let $W, V$ be two semigroup words over the set $Q_{1}=\left\{a_{1}, b_{1}, i d_{1}\right\}$. Suppose that $W$ and $V$ are reduced and $W \neq V$. We have to show that these words define different elements in the group $G\left(A_{1}\right)$. If both these words are non-empty, then we can assume that one of them (suppose $W$ ) begins with $a_{1}$ and the second begins with $b_{1}$. But then, by an easy inductive argument on the lengths of these words, we can show with the wreath recursions (3) that for every $i \geq 1$ the section $W_{\left\{w_{i}\right\}}$ begins with $a_{i+1}$ and the section $V_{\left\{w_{i}\right\}}$ begins with $b_{i+1}$, where $w_{i}=x_{1} x_{2} \ldots x_{i}$. Since the sections $W_{\left\{w_{i}\right\}}$ and $V_{\left\{w_{i}\right\}}(i \geq 1)$ are semigroup words over $Q_{i}$, we see by Proposition 8 that there is $i \geq 1$ such that $W_{\left\{w_{i}\right\}}=a_{i+1}$ and $V_{\left\{w_{i}\right\}}=b_{i+1}$. Since $a_{i+1} \neq b_{i+1}$, the words $W$ and $V$ cannot define the same element. If one of these semigroup words is empty, then the second is non-empty, i.e., it begins with $a_{1}$ or with $b_{1}$ and as above we see that they do not define the same element.

Corollary 4 The group $G(\mathcal{A})$ is of exponential growth.

Proposition 10 The automaton $\mathcal{A}$ has disappearing group words of unbounded depth.

Proof For any two integers $N, N^{\prime}$ and for $i \geq 1$ let $W\left(N, N^{\prime}, i\right)$ be a group word over the set $Q_{i}=\left\{a_{i}, b_{i}, i d_{i}\right\}$ defined as follows:

$$
W\left(N, N^{\prime}, i\right)=\left[b_{i}^{N^{\prime}} a_{i}^{-N} b_{i}^{-N^{\prime}}, a_{i}^{N}\right]:=b_{i}^{N^{\prime}} a_{i}^{N} b_{i}^{-N^{\prime}} a_{i}^{-N^{N}} b_{i}^{N^{\prime}} a_{i}^{-N} b_{i}^{-N^{\prime}} a_{i}^{N} .
$$

We see that $W\left(N, N^{\prime}, i\right)$ is a reduced group word if and only if it is a non-empty group word if and only if both $N$ and $N^{\prime}$ are non-zero integers.

For each $i \geq 1$, we consider the decompositions of the permutations $\alpha_{i}$ and $\beta_{i}$ into the products of disjoint cycles. In the decomposition of $\alpha_{i}$ (resp. $\beta_{i}$ ) let $S_{i}$ (resp. $S_{i}^{\prime}$ ) be the support of the cycle which contains the letter $x_{i}$. Let $l_{i}=\left|S_{i}\right|, l_{i}^{\prime}=\left|S_{i}^{\prime}\right|$ be the lengths of these two cycles. If $N, N^{\prime}$ are nonzero integers divisible by $l_{i}$ and $l_{i}^{\prime}$, respectively, then directly with the wreath recursions (3), we see that root permutations of elements defined by the group words $a_{i}^{N}$ and $b_{i}^{N^{\prime}}$ are trivial and the sections of these group words at any letter $x \in X_{i}$ satisfy

$$
\left(a_{i}^{N}\right)_{\{x\}}=\left\{\begin{array}{ll}
a_{i+1}^{N / l_{i}}, & x \in S_{i}, \\
\varepsilon, & \text { otherwise, }
\end{array} \quad\left(b_{i}^{N^{\prime}}\right)_{\{x\}}= \begin{cases}b_{i+1}^{N^{\prime} / l_{i}^{\prime}}, & x \in S_{i}^{\prime}, \\
\varepsilon, & \text { otherwise }\end{cases}\right.
$$

Since $S_{i} \cap S_{i}^{\prime}=\left\{x_{i}\right\}$, we obtain in this case that the root permutation of the element defined by the group word $W\left(N, N^{\prime}, i\right)$ is trivial and the section of this group word at any letter $x \in X_{i}$ satisfies

$$
\left(W\left(N, N^{\prime}, i\right)\right)_{\{x\}}= \begin{cases}W\left(N / l_{i}, N^{\prime} / l_{i}^{\prime}, i+1\right), & x=x_{i}, \\ \varepsilon, & \text { otherwise. }\end{cases}
$$

Further, for any letter $x \in X_{i}$, the section $\left(b_{i} a_{i}^{ \pm l_{i}} b_{i}^{-1}\right)_{\{x\}}$ satisfies

$$
\left(b_{i} a_{i}^{ \pm l_{i}} b_{i}^{-1}\right)_{\{x\}}=\left(b_{i}\right)_{\{x\}}\left(a_{i}^{ \pm l_{i}}\right)_{x^{\beta_{i}}}\left(b_{i}^{-1}\right)_{\left\{x_{i}\right\}}=\left(b_{i}\right)_{\{x\}}\left(a_{i}^{ \pm l_{i}}\right)_{x^{\beta_{i}}}\left(\left(b_{i}\right)_{\{x\}}\right)^{-1} \text {. }
$$


If $x=x_{i}$, then $x^{\beta_{i}}=x_{i}^{\beta_{i}} \notin S_{i}$, and if $x \neq x_{i}$, then $\left(b_{i}\right)_{\{x\}}=\varepsilon$. Thus, for any letter $x \in X_{i}$, we obtain

$$
\left(b_{i} a_{i}^{ \pm l_{i}} b_{i}^{-1}\right)_{\{x\}}= \begin{cases}a_{i+1}^{ \pm 1}, & x \in\left(S_{i}\right)^{\beta_{i}^{-1}} \\ \varepsilon, & \text { otherwise }\end{cases}
$$

and also

$$
\left(b_{i} a_{i}^{ \pm l_{i}} b_{i}^{-1}\right)(\epsilon)=i d
$$

Consequently, the section of the group word

$$
W\left(l_{i}, 1, i\right)=\left[b_{i} a_{i}^{-l_{i}} b_{i}^{-1}, a_{i}^{l_{i}}\right]=b_{i} a_{i}^{l_{i}} b_{i}^{-1} a_{i}^{-l_{i}} b_{i} a_{i}^{-l_{i}} b_{i}^{-1} a_{i}^{l_{i}}
$$

at any letter $x \in X_{i}$ is empty and the root permutation of the element defined by this group word is trivial. By the above, we see that for every $i \geq 1$ the group word $W\left(N_{i}, N_{i}^{\prime}, 1\right) \in F\left(Q_{1}\right)$, where $N_{i}=l_{1} l_{2} \ldots l_{i+1}$ and $N_{i}^{\prime}=l_{1}^{\prime} l_{2}^{\prime} \ldots l_{i}^{\prime}$, is disappearing and for the depth of this group word we have $\lambda\left(W\left(N_{i}, N_{i}^{\prime}, 1\right)\right)=i+1$.

As a direct Corollary of Propositions 8, 10 and Corollary 3, we obtain Theorem 3.

Proposition 11 (i) The automaton $\mathcal{A}$ is self-replicating.

(ii) The automaton $\mathcal{A}$ is branching over the sequence $\left(K_{i}\right)_{i \geq 1}$ of commutator subgroups $K_{i}=G\left(\mathcal{A}_{i}\right)^{\prime}$.

Proof (i) For $i \geq 1$ let $X_{i}=\left\{x_{i, 1}, x_{i, 2}, \ldots, x_{i, m_{i}}\right\}$, where, according to our assumption, we have $x_{i, 1}=x_{i}$. Let $l_{i}, l_{i}^{\prime}$ be the integers defined as in the proof of Proposition 10 . Let us fix $i \geq 1$. Since $a_{i}^{l_{i}}, b_{i}^{l_{i}^{\prime}} \in S t_{G\left(\mathcal{A}_{i}\right)}(1)$ and $\left(a_{i}^{l_{i}}\right)_{\left\{x_{i}\right\}}=a_{i+1},\left(b_{i}^{l_{i}^{\prime}}\right)_{\left\{x_{i}\right\}}=$ $b_{i+1}$, we obtain

$$
\Phi_{i, 1}\left(S t_{G\left(\mathcal{A}_{i}\right)}(1)\right)=\left\langle a_{i+1}, b_{i+1}\right\rangle=G\left(\mathcal{A}_{i+1}\right) .
$$

Since the group $H_{i}=\left\langle\alpha_{i}, \beta_{i}\right\rangle$ is transitive, for every $r \in\left\{1,2, \ldots, m_{i}\right\}$ there is $g_{r} \in$ $G\left(A_{i}\right)$ such that $x_{i, r}^{g_{r}}=x_{i}$. For every $h \in S t_{G\left(\mathcal{A}_{i}\right)}(1)$ and every $r \in\left\{1,2, \ldots, m_{i}\right\}$, we also have $g_{r} h g_{r}^{-1} \in S t_{G\left(\mathcal{A}_{i}\right)}(1)$ and

$$
\Phi_{i, r}\left(g_{r} h g_{r}^{-1}\right)=\left(g_{r} h g_{r}^{-1}\right)_{\left\{x_{i, r}\right\}}=g_{r}^{\prime} h_{\left\{x_{i}\right\}} g_{r}^{\prime-1},
$$

where $g_{r}^{\prime}=\left(g_{r}\right)_{\left\{x_{i, r}\right\}}$. By (26) we have

$$
\left\{g_{r}^{\prime} h_{\left\{x_{i}\right\}} g_{r}^{\prime-1}: h \in S t_{G\left(\mathcal{A}_{i}\right)}(1)\right\}=g_{r}^{\prime} \Phi_{i, 1}\left(S t_{G\left(\mathcal{A}_{i}\right)}(1)\right) g_{r}^{\prime-1}=G\left(\mathcal{A}_{i+1}\right)
$$

Consequently $\Phi_{i, r}\left(S t_{G\left(\mathcal{A}_{i}\right)}(1)\right)=G\left(\mathcal{A}_{i+1}\right)$ for every $r \in\left\{1,2, \ldots, m_{i}\right\}$. 
(ii) Let us fix $i \geq 1$. The commutator $\left[a_{i}^{l_{i}}, b_{i}^{l_{i}^{\prime}}\right] \in K_{i}$ belongs to the stabilizer $S t_{G\left(\mathcal{A}_{i}\right)}(1)$ and for every letter $x \in X_{i}$ the section of this commutator at the letter $x$ is empty, unless $x=x_{i}$, where we have:

$$
\left[a_{i}^{l_{i}}, b_{i}^{l_{i}^{\prime}}\right]_{\left\{x_{i}\right\}}=\left[a_{i+1}, b_{i+1}\right]
$$

The commutator subgroup $K_{i+1}=G\left(\mathcal{A}_{i+1}\right)^{\prime}$ is generated by the conjugates

$$
g^{-1}\left[a_{i+1}, b_{i+1}\right] g, \quad g \in G\left(\mathcal{A}_{i+1}\right) \text {. }
$$

Since the automaton $\mathcal{A}$ is self-replicating, we see that the preimage

$$
\Psi_{i}^{-1}\left(K_{i+1} \times\{i d\} \times \ldots \times\{i d\}\right)
$$

is a subgroup of the group $K_{i}$. Next, as in (i), we use the fact that the group $H_{i}=\left\langle\alpha_{i}, \beta_{i}\right\rangle$ is transitive and we show that for every $r \in\left\{1,2, \ldots, m_{i}\right\}$, the preimage

$$
\Psi_{i}^{-1}\left(\{i d\} \times \ldots \times K_{i+1} \times \ldots \times\{i d\}\right)
$$

is a subgroup of the group $K_{i}$, where the position of the factor $K_{i+1}$ in the above direct product corresponds to the letter $x_{i, r}$. Consequently, we obtain that the preimage

$$
\Psi_{i}^{-1}\left(K_{i+1}^{m_{i}}\right)=\Psi_{i}^{-1}\left(K_{i+1} \times \ldots \times K_{i+1}\right)
$$

is a subgroup of the group $K_{i}$.

Corollary 5 The group $G(\mathcal{A})$ is weakly branch, and in the regular case this group is self-replicating, contracting and regular weakly branch over the commutator subgroup.

Open Access This article is distributed under the terms of the Creative Commons Attribution License which permits any use, distribution, and reproduction in any medium, provided the original author(s) and the source are credited.

\section{References}

1. Bartholdi, L.: A Wilson group of non-uniformly exponential growth. C. R. Math. Acad. Sci. Paris 336, 549-554 (2003)

2. Bartholdi, L., Grigorchuk, R., Sunik, Z.: Branch groups. Handbook of algebra, vol. 3, pp. 989-1112. North-Holland, Amsterdam (2003)

3. Bartholdi, L., Kaimanovich, V.A., Nekrashevych, V.: On amenability of automata groups. Duke Math. J. 154(3), 575-598 (2010)

4. Bhattacharjee, M.: The probability of generating certain profinite groups by two elements. Isr. J. Math. 86, 311-329 (1994)

5. Bondarenko, I.: Finite generation of iterated wreath products. Arch. Math. 95, 301-308 (2010) 
6. Brieussel, J.: Amenability and non-uniform growth of some directed automorphism groups of a rooted tree. Math. Zeit. 263(2), 265-293 (2009)

7. Dey, I.M.S., Wiegold, J.: Generators for alternating and symmetric groups. J. Aust. Math. Soc. 12, 63-68 (1971)

8. Conder, M.: The symmetric genus of the Mathieu groups. Bull. London Math. Soc. 23, 445-453 (1991)

9. Erschler, A.: Piecewise automatic groups. Duke Math. J. 134(3), 591-613 (2006)

10. Erschler, A.: Automatically presented groups. Groups Geom. Dyn. 1, 47-59 (2007)

11. Feit, W.: Some consequences of the classification of finite simple groups. In: The Santa Cruz conference on finite groups, Proc. Sympos. Pure Math. 37 (American Mathematical Society), pp. 175-181 (1980)

12. Garbe, D.: Über eine Klasse von arithmetisch definierbaren Normalteilern der Modulgruppe. Math. Ann. 235, 195-215 (1978)

13. Grigorchuk, R.: Branch groups. Math. Notes 67, 718-723 (2000)

14. Grigorchuk, R., Nekrashevych, V., Sushchanskyy, V.: Automata, dynamical systems and groups. Proc. Steklov Inst. Math. 231, 128-203 (2000)

15. Gupta, N.D., Sidki, S.N.: Some infinite p-groups. Algebra i Logika 22(5), 584-589 (1983) (in Russian); Engl. transl., Algebra Log. 22(5), 421-424 (1983)

16. Guralnick, R.M., Kantor, W.M.: Probabilistic generation of finite simple groups. J. Algebra 234, 743792 (2000)

17. Jones, G.A.: Cyclic regular subgroups of primitive permutation groups. J. Group Theory 5, 403-407 (2002)

18. Macbeath, A.M.: Generators of the linear fractional groups. In: Number Theory, Proc. Symposia in Pure Mathematics, vol. 12, pp. 14-32. American Mathematical Society, Providence, RI (1969)

19. Mikolajczak, B.: Algebraic and Structural Automata Theory. Translated from the Polish Annals of Discrete Mathematics, vol. 44. North-Holland Publishing Co, Amsterdam (1991)

20. Nekrashevych, V.: Self-similar Groups. (Am. Math. Soc., Providence, RI), Math. Surv. Monogr. 117 (2005)

21. Nekrashevych, V.: Free subgroups in groups acting on rooted trees. Groups Geom. Dyn. 4, 847-862 (2010)

22. Quick, M.: Probabilistic generation of wreath products of non-abelian finite simple groups. Commun. Algebra 32(12), 4753-4768 (2004)

23. Segal, D.: The finite images of finitely generated groups. Proc. Lond. Math. Soc. 3(82), 597-613 (2001)

24. Stein, A.: $1 \frac{1}{2}$-generation of finite simple groups. Contrib. Algebra Geom. 39(2), 349-358 (1998)

25. Taylor, DE.: Pairs of generators for matrix groups I. The Cayley Bulletin, 3, 76-85. http://www.maths. usyd.edu.au:8000/u/don (1987)

26. Wilson, J.S.: Further groups that do not have uniformly exponential growth. J. Algebra 279, 292-301 (2004)

27. Woryna, A.: On permutation groups generated by time-varying Mealy automata. Publ. Math. Debrecen 67(1-2), 115-130 (2005)

28. Woryna, A.: On generation of wreath products of cyclic groups by two state time varying Mealy automata. Int. J. Algebra Comput. 16(2), 397-415 (2006)

29. Woryna, A.: The generalized dihedral groups $\operatorname{Dih}\left(\mathbb{Z}^{n}\right)$ as groups generated by time-varying automata. Algebra Discrete Math. 3, 98-111 (2008)

30. Woryna, A.: The concept of duality for automata over a changing alphabet and generation of a free group by such automata. Theor. Comput. Sci. 412(45), 6420-6431 (2011)

31. Woryna, A.: Automaton ranks of some self-similar groups, vol. 7183/2012, pp. 514-525. LNCS, (2012)

32. Woryna, A.: The concept of self-similar automata over a changing alphabet and lamplighter groups generated by such automata. Theor. Comput. Sci. 482, 96-110 (2013) 